\title{
A recombinant chimeric protein specifically induces mutant KRAS degradation and potently inhibits pancreatic tumor growth
}

\author{
Ting Pan ${ }^{1,2,3}$, Yiwen Zhang ${ }^{1,2,3}$, Nan Zhou ${ }^{1,2,3}$, Xin $\mathrm{He}^{1,2,3}$, Cancan Chen ${ }^{1,2,3}$, Liting \\ Liang $^{1,3}$, Xiaobing Duan ${ }^{1,2}$,Yingtong Lin ${ }^{1,3}$, Kang $\mathbf{W u}^{1,2,3}$, Hui Zhang ${ }^{1,2,3}$ \\ ${ }^{1}$ Institute of Human Virology, Sun Yat-sen University, Guangzhou, Guangdong, China \\ ${ }^{2}$ Key Laboratory of Tropical Diseases Control, Ministry of Education, Zhongshan School of Medicine, Sun Yat-Sen University, \\ Guangzhou, Guangdong, China \\ ${ }^{3}$ Guangdong Engineering Research Center for Antimicrobial Agent and Immunotechnology, Zhongshan School of Medicine, \\ Sun Yat-sen University, Guangzhou, Guangdong, China
}

Correspondence to: Hui Zhang, email: zhangh92@mail.sysu.edu.cn

Keywords: pancreatic cancer, Vif, Ras binding domain, ubiquitin, KRAS

Received: March 01, 2016 Accepted: June 03, $2016 \quad$ Published: June 14, 2016

\section{ABSTRACT}

Pancreatic cancer is one of the most lethal human diseases, with an all-stage 5 -year survival rate below $5 \%$. To date, no effective and specific therapy is available for this disease. Mutations in KRAS are frequently reported in pancreatic and many other cancers; thus, KRAS is an attractive therapeutic target. Our objective was to specifically eliminate mutant KRAS and induce cell death of tumors expressing this mutant protein. We thus constructed several chimeric proteins by connecting the C-terminal domains of several adaptor proteins of E3 ubiquitin ligases such as CBL, CHIP, E6AP, and VHL, as well as VIF encoded by human immunodeficiency virus type 1 (HIV-1), to the Ras binding domain (RBD) of Raf. Although all of these chimeric proteins caused the degradation of mutant KRAS and the death of KRAS-mutanttumor cell lines, the RBD-VIF with a protein transduction domain (PTD), named PTD-RBD-VIF, had the strongest tumor-killing effect. Intraperitoneally administered recombinant PTD-RBD-VIF potently inhibited the growth of xenografted KRAS-mutant pancreatic cancer cells. Our findings indicate that recombinant PTD-RBD-VIF, a chimeric protein with a combined cellular-viral origin, could be further developed for the treatment of various tumors harboring mutant or over-activated KRAS, especially for cases presenting with pancreatic cancer recurrence after surgery.

\section{INTRODUCTION}

Pancreatic carcinoma is an aggressive cancer and early diagnosis and radical surgery provide the only chance of long-term survival for patients [1-3]. A important feature of this cancer is that most pancreatic cancer cells harbor oncogenic mutations in the KRAS gene in the early stage, and the mutant KRAS is required to initiate pancreatic carcinoma $[4,5]$. Substantial evidence has demonstrated the complexity of oncogenic KRAS signaling in promoting pancreatic cancer $[6,7]$. Besides, many other tumors harbor mutations in the KRAS gene. In pancreatic cancer, colon cancer, and non-small cell lung cancer, the KRAS mutation rates are $90 \%, 45 \%$, and $35 \%$, respectively $[8,9]$. Normal KRAS proteins function as molecular switches that cycle between the GDP-bound inactive and the GTP-bound active forms [10]. When bound to GTP, they interact with the downstream protein Raf and transduce the signal to activate various cell activities such as proliferation, differentiation, apoptosis, and migration $[11,12]$. Specific point mutations in KRAS, especially those at position 12, maintain KRAS in its GTP-bound active form and consequently lead to tumor formation [13]. A variety of studies have been focused on the novel anti-kinase agents, which target the downstream kinases of KRAS signaling pathways such as MEK, PI3K and AKT [14-16]. Some of the inhibitors for these kinases are in clinical trials. However, the direct therapeutic agents for the inhibition of mutant KRAS is rare and urgently needed for the treatment of this disease. 
Ubiquitination plays a critical role in numerous biological functions [17]. It involves three enzymes: $\mathrm{E} 1, \mathrm{E} 2$ and E3. Ubiquitin is activated by E1 and is transferred to the E2. E3 recognizes the protein substrates and brings them to E2, resulting in the ubiquitination of the target protein. Subsequently, the ubiquitinated protein is recognized and degraded by the $26 \mathrm{~S}$ proteasome [18]. In humans, $2 \mathrm{E} 1 \mathrm{~s}$, approximately $50 \mathrm{E} 2 \mathrm{~s}$, and more than $600 \mathrm{E} 3 \mathrm{~s}$ have been identified to date. E3s are categorized into 2 major types [19, 20]. One contains the HECT (Homologous to E6-AP C Terminus) domain and the other contains a RING (Really Interesting New Gene) finger domain or U-box domain, which is a modified RING motif without the full complement to $\mathrm{Zn}^{2+}$-binding ligands [19, 21]. These E3s act as adaptor molecules and participate in a variety of cellular functions [20]. Some E3s could be a part of fusion protein to retarget some important proteins and mediate their degradation [22-24]. Meanwhile, the application of the knockout system at protein level with an engineered E3 ubiquitin-protein ligase has been extensively explored [25-27].

The Ras binding domain (RBD) of Raf binds quite tightly to the GTP-bound form of Ras, whereas its affinity for Ras-GDP is three orders of magnitude lower [28, 29]. Therefore, the minimal RBD of Rafl (aa 51-131) could serve as an activation-specific adaptor for mutant KRAS [30]. In this study, we screened a series of chimeric proteins by connecting the RBD domain with E3 adaptor proteins for specifically ubiquitinating and degrading mutant KRAS and found a novel therapeutic recombinant chimeric protein for targeting KRAS-mutant tumors, which can be particularly beneficial for the patients with pancreatic cancer.

\section{RESULTS}

\section{Different chimeric proteins significantly inhibit the expression of mutant KRAS}

In order to develop a therapeutic chimeric protein containing the RBD to knock down mutant KRAS, we selected several E3 ubiquitin ligases or adaptors. These included the ubiquitin ligase E6 associated protein (E6AP) encoded by the UBE3A gene, which belongs to HECT family and interacts with the E6 protein of human papillomavirus types 16 and 18, resulting in ubiquitination and proteolysis of tumor protein p53 [31]; Casitas B-lineage lymphoma proto-oncogene (CBL), a proto-oncogene that encodes a RING finger E3 ligase [32]; and carboxyl-terminus heat shock cognate 70 -interacting protein (CHIP), with a C-terminal U-box domain, which is known to ubiquitinate short-lived proteins [33]. Of the well-known adaptors of E3 ligase, we selected von Hippel-Lindau syndrome (VHL) and
HIV-1 virion infectivity factor (VIF), which are adaptors for the complex including elongin $\mathrm{B}$, elongin $\mathrm{C}$, and cullin-2/5 that possesses E3 ubiquitin ligase activity $[34,35]$. The protein levels of these various chimeric plasmids were confirmed by western blot. As shown in Figure 1A, the RBD of Raf-1, which has been reported to bind to mutant KRAS, was chosen as the KRASbinding domain for the chimeric proteins. To determine whether these chimeric proteins could degrade mutant KRAS, a plasmid encoding mutant KRAS-RFP fusion protein was constructed and used for quantification of the KRAS level in HEK293T cells. After co-transfection of the mutant KRAS-RFP-harboring plasmids and the plasmids encoding various chimeric proteins, we found that the cellular KRAS-RFP levels, determined by fluorescence microscopy, were significantly decreased by these chimeric proteins, especially by RBD-CHIP and RBD-VIF (Figure 1B). The levels measured by mean fluorescent intensity (MFI) also showed similar results (Figure 1C). Western blotting revealed that the expression of mutant KRAS ${ }^{\mathrm{G} 12 \mathrm{D}}$ or $\mathrm{KRAS}^{\mathrm{G} 12 \mathrm{~V}}$ was also potently inhibited by these chimeric proteins in a dosedependent manner (Figure 1D).Besides, the chimeric RBD-VIF have no effect on the degradation of wild-type KRAS (Supplementary Figure S1).

\section{Recombinant chimeric proteins induce cell death in mutant KRAS-expressing tumor cell lines}

We then examined the specific degradation of mutant KRAS by the recombinant chimeric proteins. To facilitate the uptake of recombinant chimeric proteins into cells, the three repeated protein transduction domains (PTDs) of HIV-1 Tat protein, which have powerful transmembrane transporting capabilities [36], were connected to the $\mathrm{N}$-termini of the chimeric proteins (Figure 2A). As the expression of recombinant PTD-RBD-CBL was not induced well by $1 \mathrm{mM}$ isopropylthio- $\beta$-d-galactoside (IPTG), we did not continue to test this chimeric protein. After expression and purification of the rest of the recombinant chimeric proteins, we examined their abilities to induce cell death in several mutant KRAS-expressing tumor cell lines. We found that almost all of the cells harboring KRAS-mutant died at $48 \mathrm{~h}$ after treatment with the recombinant chimeric proteins, whereas the growth of non- KRAS mutant cell lines, such as HEK293T cells and Bxpc-3 cells, were not inhibited. Quantification of cell death showed the similar results (Figure 2B and SupplementaryFigure S2). PTDRBD-VIF and PTD-RBD-CHIP exhibited high efficiency to specifically induce the death of Panc-1 cells (Figure 2C). Furthermore, the IC50 of PTD-RBD-CHIP, PTD-RBDE6AP, PTD-RBD-VHL and PTD-RBD-VIF in the Panc-1 cell line was $89 \mu \mathrm{M}, 6 \mathrm{mM}, 20 \mathrm{mM}$ and $5 \mu \mathrm{M}$, respectively, as measured by flow cytometry. Thus, PTD-RBD-VIF showed the best capacity to induce cell death (Figure 2D). 


\section{Evaluation of immunogenicity and safety of purified proteins}

Considering the possible development of protein inhibitors for pancreatic cancer, it is important to determine the immunogenicity and safety of the recombinant chimeric proteins. To this end, 4-week-old female $\mathrm{BALB} / \mathrm{c}$ mice were immunized subcutaneously with $1 \mu \mathrm{g}$ of PTD-RBD-VIF or PTD-RBD-CHIP. PTDRBD-VIF exhibited considerably low immunogenicity (Figure 3A). To determine the toxicity, the acute toxicities of PTD-RBD-VIF on mice were tested. Two weeks after intraperitoneal injection of PTD-RBD-VIF at $0 \mathrm{mg} / \mathrm{kg}, 10 \mathrm{mg} / \mathrm{kg}$, or $20 \mathrm{mg} / \mathrm{kg}, 5 \mathrm{male}$ BALB $/ \mathrm{c}$ mice did not show significant histological changes in heart, liver, lung, spleen, and kidney in HE staining (Figure 3B). Additionally, no significant abnormality was found in body weight (Figure 3C). The functions of key enzymes, including AST, ALT, BUN, and CRE, in the histological sections of these organs were also normal ranges (Figure 3D). Collectively, recombinant PTDRBD-VIF had the highest efficiency to specifically induce the degradation of mutant KRAS and the death of cells harboring mutant KRAS, with low immunogenicity and tolerable toxicity in mice.

\section{RBD-VIF mediates the ubiquitination and degradation of mutant KRAS and inhibits the downstream of MAPK-ERK pathway}

To investigate the mechanism underlying the specific degradation of KRAS by RBD-VIF, the HA-tagged RBDVIF and mutant KRAS plasmids were co-transfected into HEK293T cells and the interaction between mutant KRAS and RBD-VIF was examined. Mutant KRAS was coimmunoprecipitated with HA-tagged RBD-VIF chimeric protein (Figure 4A). To further confirm the involvement of the ubiquitin system in RBD-VIF-mediated KRAS degradation, we treated the transfected Panc-1 cells with MG-132, a specific inhibitor of the 26S proteasome. The levels of mutant KRAS were restored in the presence of MG-132 (Figure 4B). It is known that Vif binds to Elongin $\mathrm{B}$, Elongin $\mathrm{C}$, and Cullin5 to form an E3 ubiquitin ligase complex resulting in the ubiquitination of $\mathrm{APOBEC} 3 \mathrm{G}$ [34]. All of the protein members of this complex are vital for its function. To determine whether or not these
A

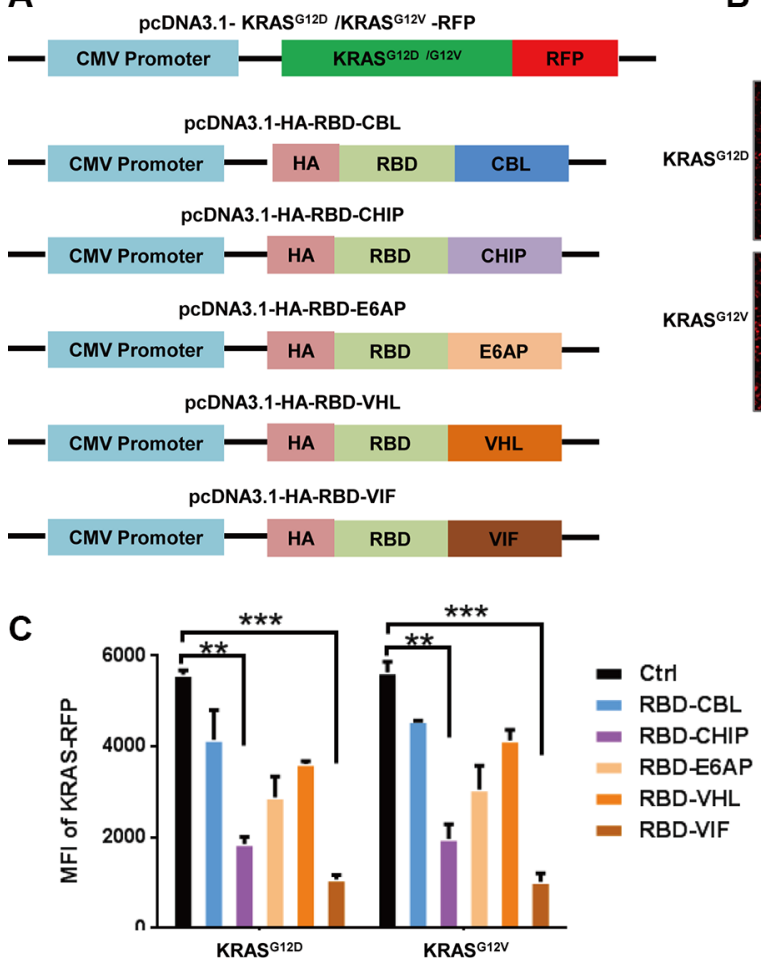

B
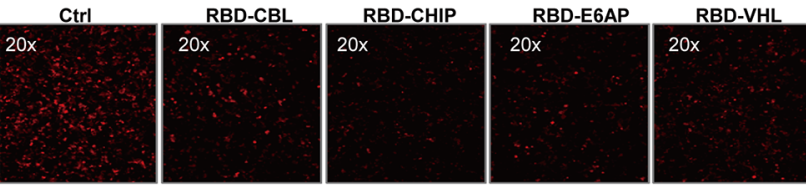

$20 x$

RBD-VIF

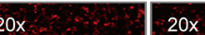

$20 x$

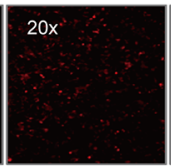

$20 x$
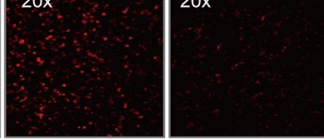

$0 \mathrm{x}$

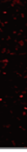

$20 x$

D

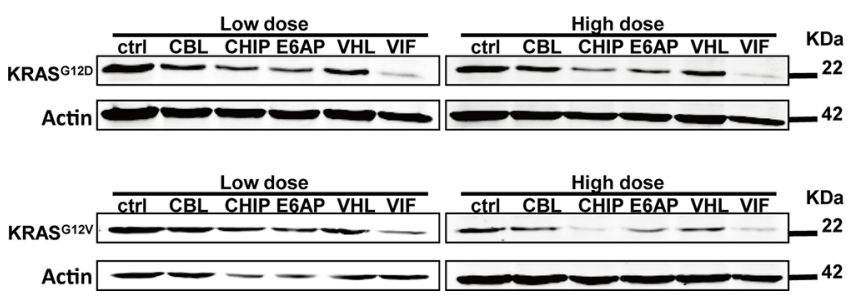

Figure 1: Different chimeric proteins significantly inhibit the expression of mutant $\operatorname{KRAS}^{\mathrm{G} 12 \mathrm{D}}$ or $\mathrm{KRAS}^{\mathrm{G} 12 \mathrm{~V}}$. (A) Schematic of construction of different chimeric proteins and mutant KRAS-RFP-harboring plasmids. (B) Different plasmids harboring various chimeric proteins were co-transfected with KRAS ${ }^{\mathrm{G} 12 \mathrm{D}}$ or KRAS ${ }^{\mathrm{G} 12 \mathrm{~V}} \mathrm{RFP}-$-expressing plasmids into HEK293T cells. After $48 \mathrm{~h}$, KRAS-RFP expression was detected under fluorescence microscope. (C) The MFI results were evaluated by flow cytometry and the mean \pm SEM are shown. Error bars indicate SEM. ${ }^{*} p<0.05,{ }^{* *} p<0.01$ compared to controls. (D) KRAS ${ }^{\mathrm{G} 12 \mathrm{D}}$ or KRAS ${ }^{\mathrm{D} 12 \mathrm{~V}}$ RFP-expressing plasmids were co-transfected with high or low doses of adaptor plasmids into HEK293T cells, and the levels of mutant KRAS were determined by western blotting after $48 \mathrm{~h}$. 
A

PTD: MGRKKRRQRRRGHSGRKKRRQRRR GHIYPYDVPDYAGDPGRKKRRQRRR

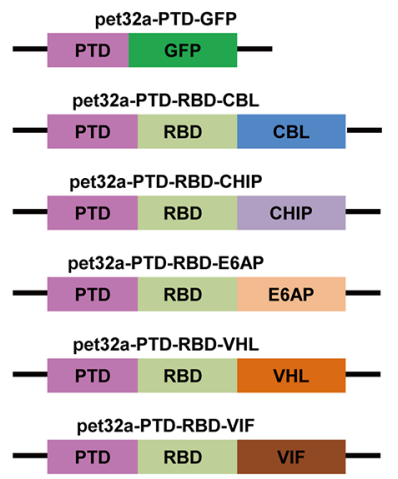

C

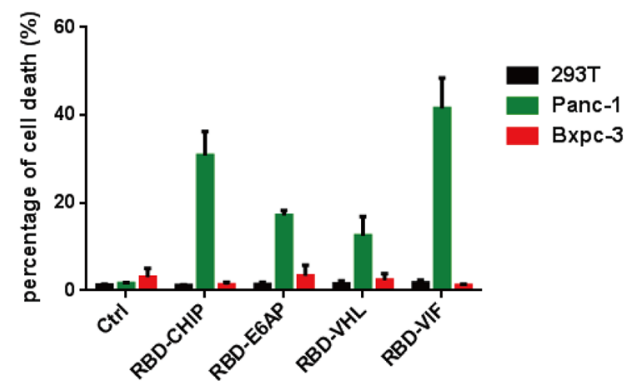

B

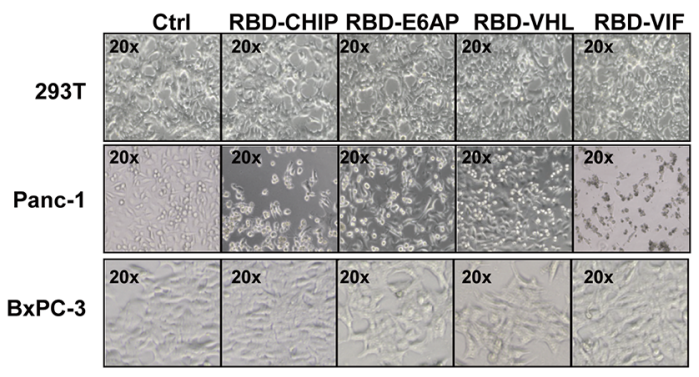

D

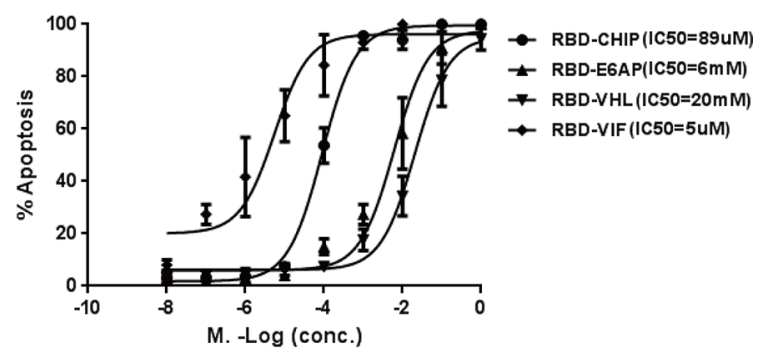

Figure 2: Purified chimeric proteins induce cell death in tumor cell lines. (A) Schematic of construction of different chimeric proteins. (B) The purified proteins were added to different cell cultures. After $48 \mathrm{~h}$, cells were examined under microscope. (C) The percentage of cell death was evaluated by MTT assay, and mean \pm SEM is shown. Error bars indicate SEM. (D) Different doses of chimeric proteins were added to Panc-1 cells and IC50 was calculated. Error bars indicate SEM.

A

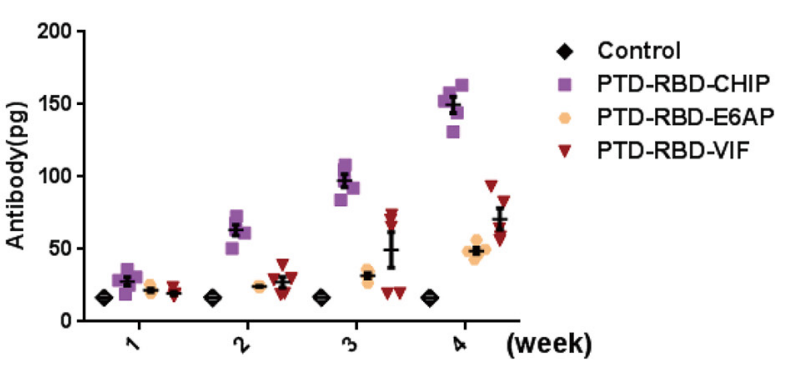

B

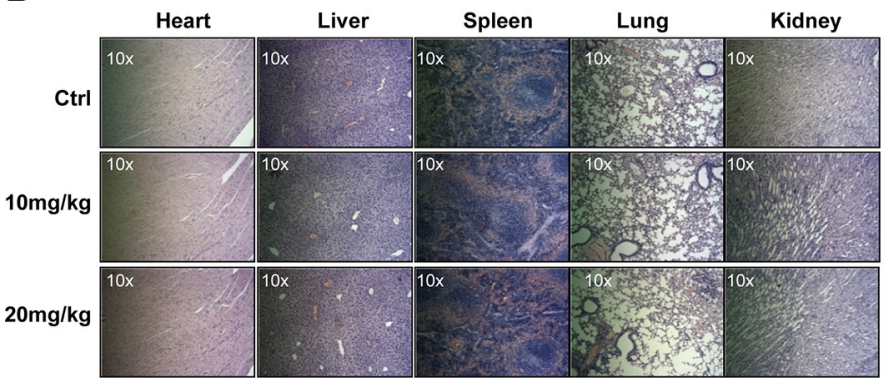

C

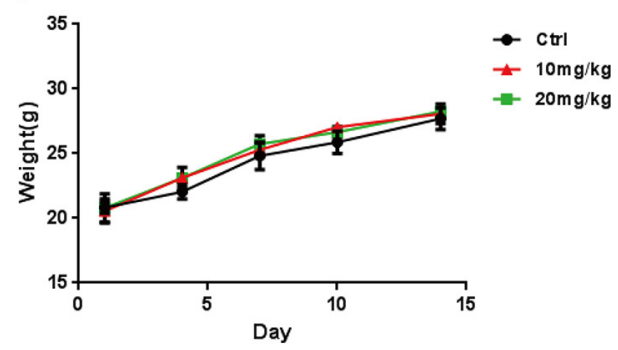

D

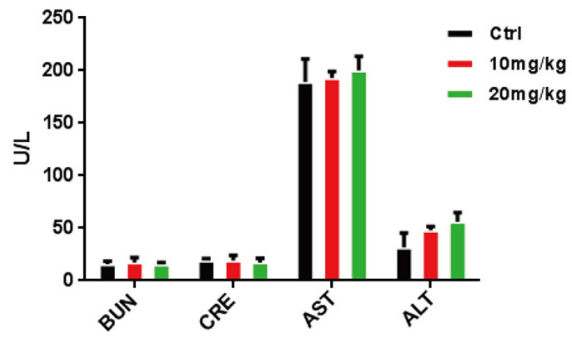

Figure 3: Purified PTD-RBD-VIF has low immunogenicity and toxicity. (A) Different chimeric proteins (PTD-RBD-CHIP, PTD-RBD-E6AP, PTD-RBD-VIF) were intraperitoneally injected into 6 mice individually and the immunogenicity of each protein was detected by ELISA after 4 wk. Error bars indicate SEM. (B-D) The safety of PTD-RBD-VIF was examined by the acute toxicity test. (B) Vital organs, including spleen, lung, liver, kidney, and heart, from mice treated with various doses of PTD-RBD-VIF were histologically sectioned and stained with HE. (C) Weight of male BALB/c mice after intraperitoneal injection of PTD-RBD-VIF. Error bars indicate SEM. (D) The effect of PTD-RBD-VIF on the hepatic and renal functions of mice. Error bars indicate SEM. (BUN: Blood urea nitrogen, CRE: Creatine, AST: Aspartate transaminase, ALT: Alanine transaminase) 
proteins are involved in the degradation of mutant KRAS, we knocked down the expression of Elongin B, Elongin $C$, and Cullin 5 using specific siRNAs. The levels of mutant KRAS-RFP were rescued by the suppression of these proteins (Figure 4C), supporting that RBD-VIF mediates the degradation of mutant KRAS through the Vif-conjugated ubiquitin system. Alternatively, because ERK1/2 and MEK are major downstream kinases in the KRAS signaling pathway $[37,38]$, we measured the level of ERK1/2 and MEK phosphorylation to explore the mechanism by which RBD-VIF suppresses mutant KRAS. As shown in Figure 4D and 4E, treatment of Panc-1 cells with recombinant PTD-RBD-VIF significantly decreased the phosphorylation of ERK1/2 and MEK. These results were consistent with a previous report that inhibition of KRAS inhibits the Raf/MEK/ERK signaling pathway [38].

\section{Different PTD-RBD-VIF administration routes for treatment of the xenograft tumors in nude mice}

To further study the in vivo anti-tumor activity of recombinant PTD-RBD-VIF, we established a Panc-1 cell xenograft tumor model in BALB/c nude mice. Each mouse was subcutaneously injected with $1 \times 10^{6}$ Panc- 1 cells at the abdomen and randomly divided into 4 groups. The mice in the experimental groups were injected with $100 \mathrm{mg}$ of PTDRBD-VIF every 3 days. The control protein, recombinant PTD-GFP, was injected into the mice in control group. As shown in Figure $5 \mathrm{~A}-5 \mathrm{C}$, the orthotopic injection was more effective than tail vein injection and intraperitoneal injection in this xenografted mouse model. Furthermore, we also established a xenograft tumor model in BALB/c nude mice with BxPC-3 cells. The mice were injected with $1 \times$ $10^{6} \mathrm{BxPC}-3$ cells at the abdomen and orthotopically treated with PTD-RBD-VIF or a control protein. As expected, PTD-RBD-VIF has no effect on pancreatic cell line without mutant KRAS (Figure 5D and 5E).

\section{Recombinant PTD-RBD-VIF protein induces tumor cell death in vivo}

Considering the anatomical location of pancreas and the actual clinical treatments for pancreatic cancer, we continued our study by using intraperitoneal injection. Again, the mice were injected intraperitoneally with $100 \mu \mathrm{g}$ of PTD-RBD-VIF or control protein PTD-GFP every 3 days. A significant difference in tumor volume was observed between the PTD-RBD-VIF-treated and control groups at days 20 to 40 after treatment (Figure 6A). The representative gross morphology of these tumors is shown in Figure 6B. Tumor weight and the survival rate of mice in the 2 groups were significantly different (Figure 6C and 6D). Furthermore, to confirm that PTDRBD-VIF inhibited the expression of mutant KRAS in vivo, representative tumor tissues were digested with collagenase and hyaluronidase and cells were disrupted and analyzed by western blotting. The expression of mutant KRA was indeed decreased (Figure 6E). TUNEL staining revealed that cell death was increased in the RBD-VIF treated group compared to the control group (Figure 6F). Taken together, our data indicate that recombinant PTD-RBD-VIF interacts with mutant KRAS and induces its ubiquitination and degradation, thereby inhibiting the growth of pancreatic cancer cells in vitro and in vivo.

\section{DISCUSSION}

KRAS has long been considered a target for cancer therapeutics. The siRNA-driven knockdown of KRAS was previously shown to cause apoptosis of KRAS mutant-expressing cancer cells [39, 40]. Although RNA interference (RNAi) provides an alternative therapeutic approach for inhibiting KRAS gene function, the efficient delivery of siRNAs remains a problem and early attempts to develop small molecules targeting KRAS as a direct pancreatic cancer therapy were not successful. Several drugs have been developed to inhibit the farnesylation of RAS, is essential for the biological activity of RAS [41, 42]. A farnesylation inhibitor, R115777, has completed a Phase II clinical trial for use in the treatment of pancreatic cancer. Another farnesylation inhibitor, anthroquinonol, is in a phase II clinical trial for non-small cell lung cancer (NSCLC). In addition, a recent report showed that an engineered ubiquitin ligase suppressed pancreatic cancer cells by targeting mutant KRAS [23]. However, these chimeric genes were carried by a lentiviral vector. Given the low delivery efficiency of recombinant lentiviral viruses into the tumor mass, this gene therapy strategy is unlikely to be further developed as a new treatment modality in the near future .

In this study, we generated a novel recombinant chimeric protein PTD-RBD-VIF for specific ubiquitination and degradation of mutant KRAS. HIV-1 Vif is a highly conserved viral protein that induces ubiquitination and degradation of host restriction factor APOBEC $3 \mathrm{G}$ $[34,43,44]$. By comparative analysis, we found that PTD-RBD-VIF is the most effective of the chimeric proteins for degradation of mutated KRAS. PTD-RBDVIF degrades KRAS through the same pathway used by VIF to degrade APOBEC3G. This was consistent with the previous finding that depletion of elongin $\mathrm{B}$, elongin $\mathrm{C}$, or Cullin5, which are components of the E3 ligase complex required for the degradation of $\mathrm{APOBEC} 3 \mathrm{G}$, decreased the degradation of mutant KRAS [34]. Although it has been reported that several other engineered E3 ligases could dregade oncoproteins such as HER2, C-MYC, and KRAS, none of them has been further developed as an effective recombinant protein for the treatment of cancer [22-24]. It could be due to their relatively low efficiency to induce the cancer cell death, as we have demonstrated in Figure 2D. 
Our study indicates that the IC50 of recombinant PTDRBD-VIF to induce cell death is $5 \mathrm{uM}$, which is much lower than that of other chimeric proteins. We are not surprised by this result because the highly-conserved nature of Vif suggests that its ubiquitination function is critical for HIV-1.

Pancreatic cancer is the fourth leading cause of cancer-related deaths and the usual survival rate of patients is under six months [3]. Currently, the routine treatment includes surgery, chemotherapy, radiotherapy, and administration of immunosuppressants [45, 46]. Even when the pancreatic tumor is surgically resected, the postsurgery survival rate is exceptionally poor. Recurrent pancreatic carcinoma remains a significant therapeutic challenge and no treatment has shown a strong impact to date [47]. Because pancreatic carcinoma recurrences after surgery resecting are mainly local retroperitoneal recurrence, quickly followed by hepatic metastases or peritoneal dissemination, it is difficult to choose secondary surgery $[48,49]$. Given the complex biology and clinical characteristics of pancreatic cancer, we designed an intraperitoneal injection to simulate the natural clinical condition, although the orthotopic injection was more effective than tail vein injection and intraperitoneal injection in our xenografted mouse model of pancreatic cancer. After direct injection of recombinant PTD-RBDVif into the abdominal cavity, the chimeric protein potently inhibited the growth of pancreatic cancer implanted subcutaneously at the abdomen, which mimicked the retroperitoneally-located pancreatic cancer. Furthermore, intraperitoneal administration of this recombinant protein was found to be safe and convenient. Our findings reveal that the recombinant PTD-RBD-VIF has potential for treatment of pancreatic carcinoma in vivo. We expect that intraperitoneal administration of this recombinant protein after surgery resecting pancreatic cancer could increase the
A

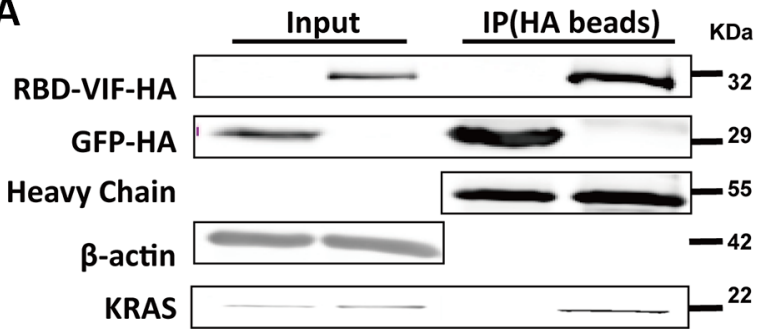

D

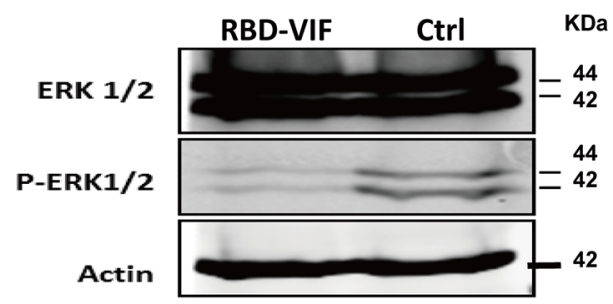

B

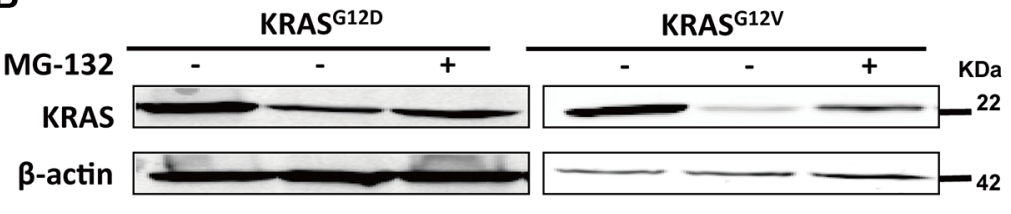

E

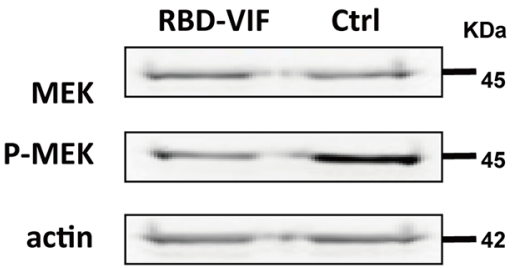

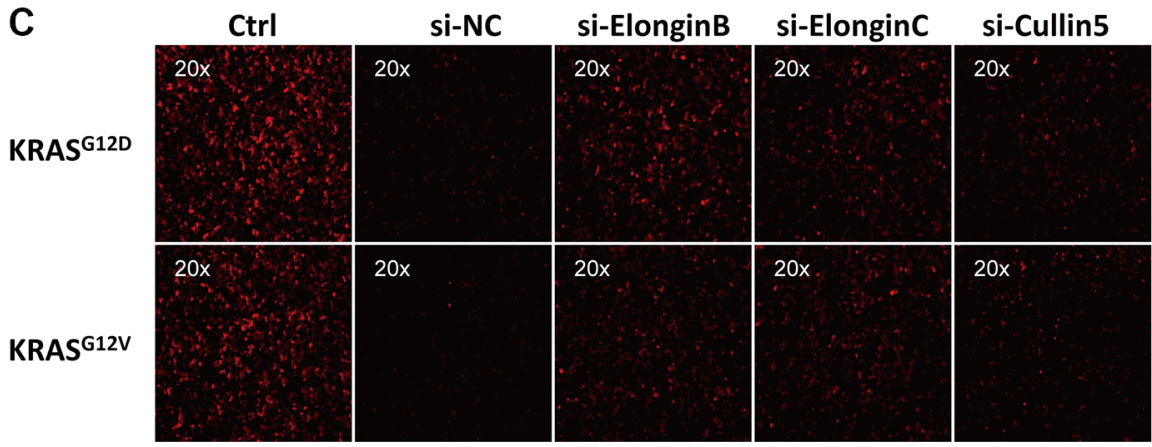

Figure 4: RBD-VIF mediates the degradation of mutant KRAS through Vif-mediated ubiquitin system and inhibits the downstream of MAPK-ERK pathway. (A) RBD-VIF-HA plasmid and KRAS plasmid were co-transfected into HEK293T cells, and $48 \mathrm{~h}$ later, cells were collected for co-immunoprecipitation experiments. GFP-HA plasmid was transfected as a negative control. (B) RBD-VIF-HA plasmid and mutant KRAS plasmid were co-transfected into HEK293T cells and the culture was maintained for $12 \mathrm{~h}$ with or without $2 \mu \mathrm{M}$ MG-132 before harvesting the cells for western blot analysis at $48 \mathrm{~h}$. (C) Mutant KRAS-RFP-expressing plasmids were co-transfected with si-NC, si-Elongin B, si-Elongin C, or si-Cullin 5 respectively into HEK293T cells. After 48 h, the expression of KRAS-RFP was detected under fluorescence microscope. (D) Panc-1 cells were treated with RBD-VIF or a control protein (PTD-GFP). After $24 \mathrm{~h}$, cells were harvested for western blot analysis to detect the phosphorylation of ERK1/2. (E) Panc-1 cells were treated with RBDVIF or the control protein PTD-GFP for $24 \mathrm{~h}$ and then the cells were harvested for western blot to detect P-MEK expression. 
A

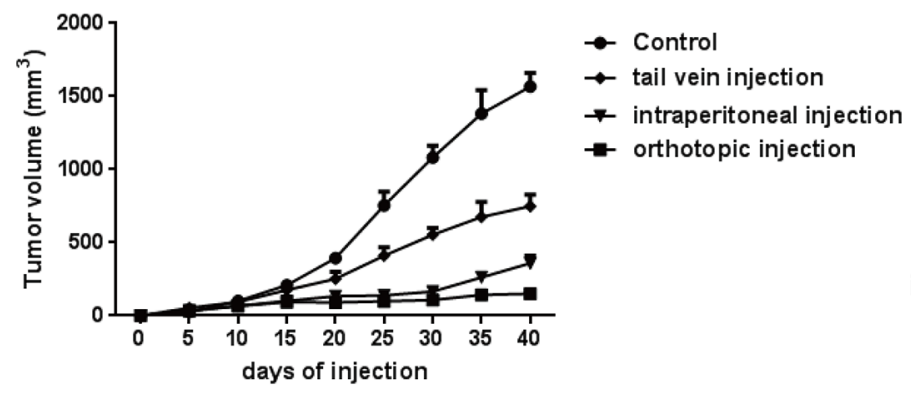

B

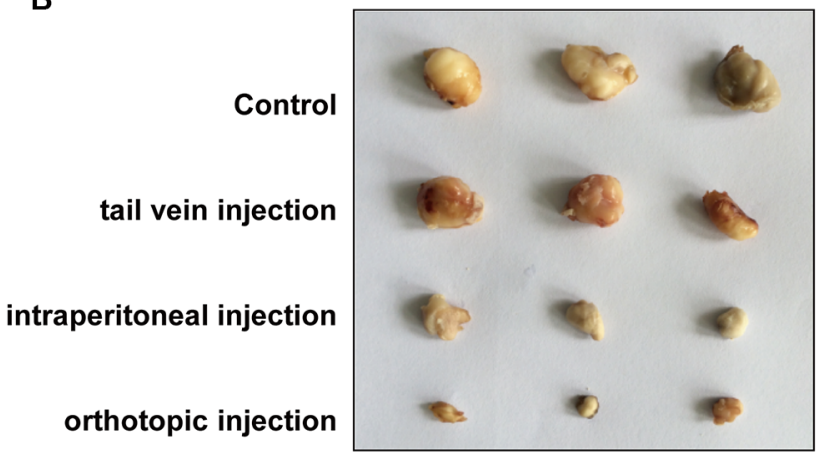

D

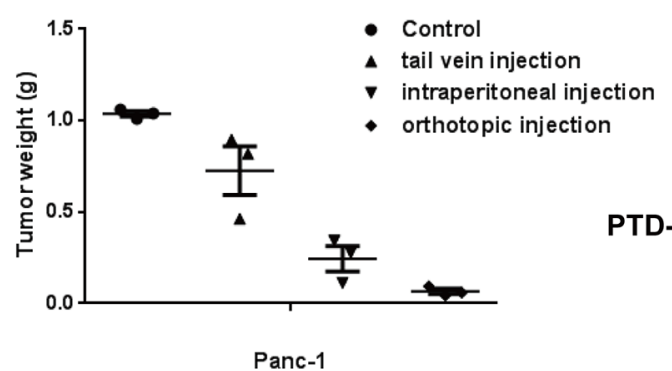

E

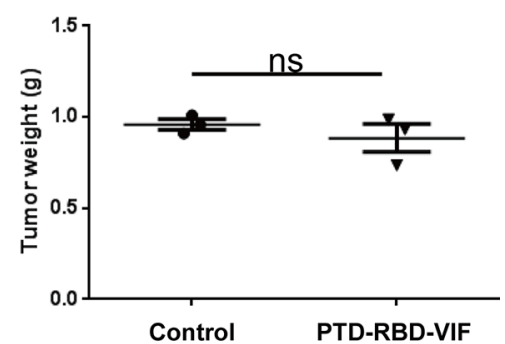

Figure 5: Comparison of PTD-RBD-VIF with different ways of administrations in nude mice. (A-C) Xenografts were established using Panc-1 cell line in female BALB/c nude mice. Four groups were randomly assigned and injected with control protein or recombinant PTD-RBD-VIF by tail vein injection, intraperitoneal injection and orthotopic injection every 3 days $(n=3)$. (A) Tumor volumes were measured every 5 days. Error bars indicate SEM. (B) Tumor formation assay to evaluate the effect of different PTD-RBDVIF delivery pathways. (C) The data represent mean \pm SEM $(n=3)$. Error bars indicate SEM. (D-E) Xenografts were established using $\mathrm{BxPC}-3$ cell line in female BALB/c nude mice. Two groups were randomly assigned and injected with control protein or recombinant PTDRBD-VIF by tail vein injection every 3 days $(n=3)$. (D) Tumor formation assay to evaluate the effect of different PTD-RBD-VIF delivery pathways. (E) The data represent mean $\pm \operatorname{SEM}(n=3)$. Error bars indicate SEM.

A

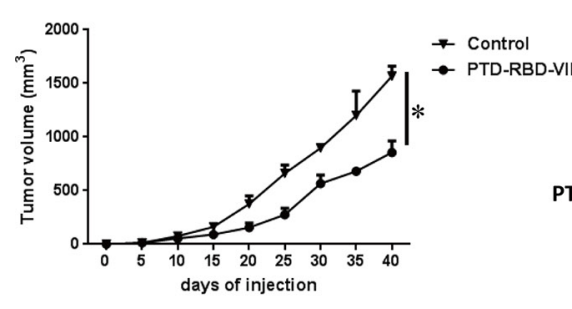

D

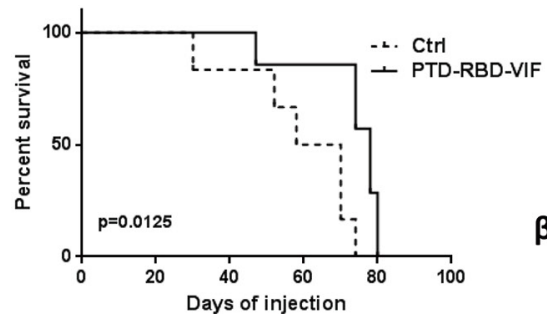

B

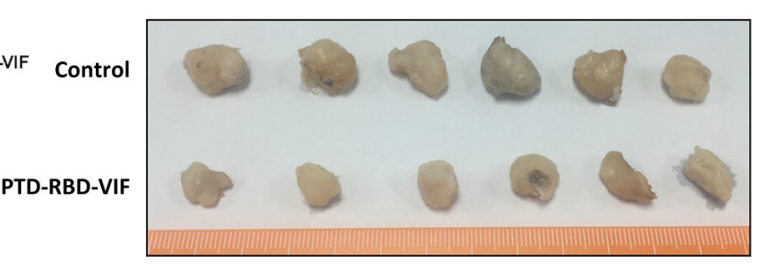

E

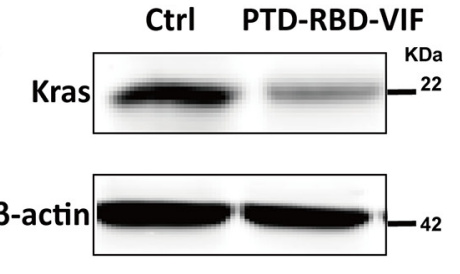

C

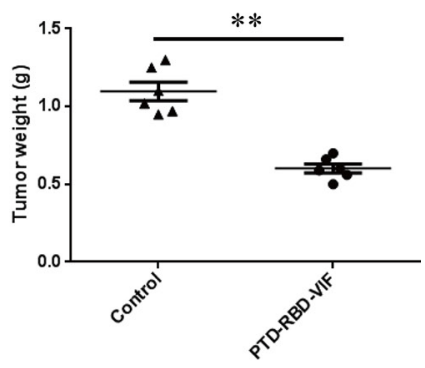

F
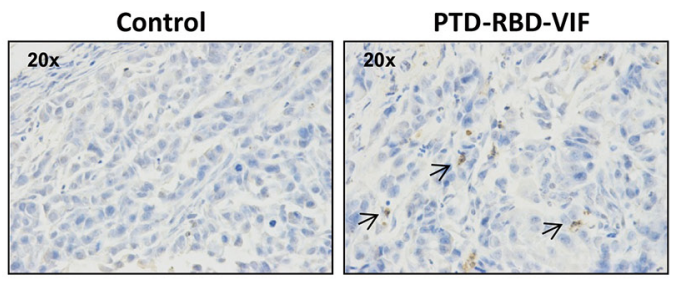

Figure 6: PTD-RBD-VIF potently inhibits pancreatic tumor growth with intraperitoneal injection in vivo. Xenografts were established using Panc-1 cell line in female BALB/c nude mice. Two groups were randomly assigned and injected (i.p.) with recombinant PTD-RBD-VIF or control protein every 3 days $(n=6)$. (A) Tumor volumes were measured every 5 days. Error bars indicate SEM. (B) Tumor formation assay to evaluate the effect of PTD-RBD-VIF or control protein. (C) The data represent mean $\pm \operatorname{SEM}(n=6)$. Error bars indicate SEM. (D) Survival curve for 2 groups of mice. (E) Representative western blot to detect the expression of KRAS in the tumors in these 2 groups. (F) Representative TUNEL staining of tumors in each group. 
survival rate and quality of life of pancreatic carcinoma patients, although further evaluation of its druggability is needed.

\section{MATERIALS AND METHODS}

\section{Cell culture and transfection}

Human Panc-1 cells, Bxpc-3 cells and HEK293T cells were obtained from ATCC and grown at $37^{\circ} \mathrm{C}$ in Dulbecco's modified Eagle's medium (Gibco) supplemented with $10 \%$ fetal calf serum (Gibco), $100 \mathrm{units} / \mathrm{ml}$ of penicillin and $100 \mu \mathrm{g} / \mathrm{ml}$ of streptomycin (Gibco). Lipofectamine 2000 (Invitrogen) was used for transfection of plasmids or si-RNAs respectively by following the instruction of manufacturer.

\section{Plasmid construction}

The wide-type KRAS DNA fragment was generated by PCR with the isolated genomic DNA of Bxpc-3 cells as the template. It was then inserted into pcDNA3.1 vectors. The G12D and G12V mutants were generated by point mutation assay, as described by us previously [50]. The sequence of Vif C-terminus was amplified with PCR from an infectious HIV-1 clone pNL4-3 and inserted into pcDNA3.1 harboring an intron. The other adaptor genes, CHIP, E6AP, CBL, VHL, were amplified from cDNA of HEK293T cells by PCR. The accuracy of all the clones was confirmed by DNA sequencing.

\section{Small interfering RNA (siRNA) knockdown}

The human Elongin B, Elongin $\mathrm{C}$ and Cullin5specific siRNAs and a negative control siRNA were purchased from Ribo, Inc. (Guangzhou, China). The HEK293T cells were transfected with $50 \mathrm{nM}$ siRNA using Lipofectamine RNAimax (Invitrogen, Inc.). After $48 \mathrm{~h}$, the knockdown efficiency were detected by qRT-PCR.

\section{Protein purification and endotoxin removal}

The plasmids pET32a harboring PTD-RBDVIF or other chimeric genes were transformed into E. Coli BL21 competent cells (Novagen) respectively. After the expression of proteins was induced by $1 \mathrm{mM}$ isopropylthio- $\beta$-d-galactoside, the bacterial cells were lysed by sonication. The insoluble fraction was pelleted at $10,000 \times \mathrm{g}$ for $10 \mathrm{~min}$, and the supernatant was applied to a Ni-conjugated agarose bead column (GE). After washing, the bound His fusion proteins were eluted with $500 \mu \mathrm{M}$ Imidazole. The proteins were then suspended in PBS buffer and the concentration was measured by the Bradford method. For the in vivo tumor model experiments, the His-tag was removed first. And then the no-tagged PTD-RBD-VIF proteins was pruified by a heparin- agarose chromatography and subsequently ion exchange [51]. The proteins were then suspended in PBS buffer and the concentration was measured by the Bradford method. The purities of expressed proteins were $>95 \%$. Moreover, the possible endotoxin had been removed from the recombinant protein with Triton X-114 as described previously and the residual endotoxin was examined with limulus amoebocyte lysate (LAL) assay (Cambrex Bio Science) by following the instruction of manufacturer [52]. The samples were then aliquoted and frozen at $-80^{\circ} \mathrm{C}$.

\section{Immunogenicity assay in mice}

All animal works were performed incompliance with the institutional guidelines and approved protocols. The 4-week-old female BALB/c mice were intradermally immunized with the recombinant PTD-RBD-VIF or PTD-RBD-CHIP protein mixed in a complete Freund's adjuvant (Sigma) at day 0 . Then these mice were boosted three times with proteins mixed in an incomplete Freund's adjuvant (Sigma) every week. Protein-specific antibody responses were measured by ELISA. Briefly, the recombinant PTD-RBD-VIF or PTD-RBD-CHIP proteins diluted in PBS were coated in 96-well plates overnight at $4^{\circ} \mathrm{C}$, followed by $30 \mathrm{~min}$ of blocking with non-fat milk. The serum samples were then added and incubated at room temperature for $1 \mathrm{~h}$. After washing, the bound antibodies were detected using the HRP-labeled goat antimouse IgG $(\mathrm{H}+\mathrm{L})$ and TBM substrate. The OD $450 \mathrm{~nm}$ was recorded and used as a relative measurement for antibody titer.

\section{Western blot and co-immunoprecipitation (Co-IP)}

HEK293T cells were transfected with chimeric adaptor-expressing plasmids and mutant KRASexpressing plasmid and were lysed $48 \mathrm{~h}$ later with lysis buffer. The lysate were then subjected to electrophoresis, followed by transferring onto the membrane and detection with the primary antibodies including anti-KRAS (mouse monoclonal, CST) or anti-beta-actin (mouse monoclonal, MBL). For the co-IP experiment, the lysate were incubated with anti-HA beads (Sigma) overnight at $4^{\circ} \mathrm{C}$. Then IP products were centrifuged and washed three times with lysis buffer. Western blot was conducted to analyze the immunoprecipitated samples with the primary antibodies including anti-KRAS (rabbit polyclonal, MBL) or anti-HA (mouse monoclonal, MBL).

\section{Cell toxicity test}

Cell toxicity assay was performed with the CellTiterGlo Luminescent Cell Viability Assay Kit ( Promega). The instructions of manufacturer were followed. Luminescence was recorded with a Promega plate reader. 


\section{Apoptosis assay}

An Annexin V-FITC Apoptosis Detection Kit (KeyGen Biotech, Nanjing, China) was used for detecting apoptosis according to the instructions of manufacturer. The Panc-1cells were labeled by Annexin-V and then were detected by a flow cytometer LSR Fortessa (Becton Dickinson).

\section{Acute toxicological assay}

Male BALB/c mice, 4-6 weeks, were purchased from Laboratory Animal Center in Sun Yat-Sen University, Guangzhou, China. These mice were randomly divided into three groups and were then intraperitoneally injected with recombinant proteins at different doses. After two weeks, mice were sacrificed. The organs including heart, liver, spleen, lung, and kidney were fixed in $4 \%$ formaldehyde at room temperature for hematoxylin and eosin staining and the blood samples were subjected to the analysis of hepatic or renal functions.

\section{In vivo experiments}

All animal procedures were conducted in accordance with the protocols generated by the Institutional Animal Care and Use Committee at the Sun Yat-Sen University. For subcutaneous xenografts, 6-week-old female BALB/c nude mice were injected with $1 \times 10^{6}$ cells in a $100 \mu \mathrm{l}$ suspension. Then these mice were randomly divided into 2 groups. One group was received the treatment of PTDRBD-VIF injection and another group was received a control protein PTD-GFP-VIF. These two groups were intraperitoneal injection with 100ug recombinant proteins every three days respectively, until they died.

\section{Statistics and graphs}

Statistical analyses were carried out using Prism software (GraphPad). All of data are reported as mean \pm SEM. Differences were found to be significant when $\mathrm{P}$ was less than 0.05 or 0.01 , as indicated by single $(*)$ or double asterisks $(* *)$ within the figures. Most graphs were produced using Prism. Flow cytometry data was processed using FlowJo (Tree Star).

\section{Abbreviations}

HIV-1: human immunodeficiency virus type 1; RBD: Ras binding domain; PTD: protein transduction domain; HECT: Homologous to E6-AP C Terminus; RING: Really Interesting New Gene; E6AP: E6 associated protein; CBL: Casitas B-lineage lymphoma proto-oncogene; CHIP: carboxyl-terminus heat shock cognate 70-interacting protein; VHL: von Hippel-Lindau syndrome; VIF: HIV-1 virion infectivity factor; IPTG: isopropylthio- $\beta$-d-galactoside; MFI: mean fluorescent intensity; RNAi: RNA interference; NSCLC: non-small cell lung cancer; BUN: Blood urea nitrogen; CRE: Creatine; AST: Aspartate transaminase; ALT: Alanine transaminase.

\section{ACKNOWLEDGMENTS}

This work was funded by National Special Research Program for the Important Infectious Diseases (No.2013ZX10001004) and Guangdong Innovative Research Team Program (No. 2009010058) to H.Z. This work was also supported by National Natural Science Foundation of China (No.301500740), Natural Science Foundation of Guangdong (No. 2015A030310442) and China Postdoctoral Science Foundation (No. 2015M580757) to T.P.

\section{CONFLICTS OF INTEREST}

The authors have declared that no conflicts of interest exists.

\section{Authors' contributions}

All listed authors contributed to this work and reviewed the manuscript. Ting Pan designed the experiments and performed most of these experiments. $\mathrm{Xin} \mathrm{He}$, Cancan Chen, Kang Wu and Yingtong Lin purified these proteins and performed the entire animal model of tumor cells. Nan Zhou, Xiaobing Duan and Liting Liang performed some molecular clone, western blot and co-IP assay. Ting Pan and Hui Zhang contributed to the idea generation, experimental design, manuscript preparation and conceived the project.

\section{REFERENCES}

1. Conroy T, Desseigne F, Ychou M, Bouche O, Guimbaud R, Becouarn Y, Adenis A, Raoul JL, Gourgou-Bourgade S, de la Fouchardiere C, Bennouna J, Bachet JB, KhemissaAkouz F, et al. FOLFIRINOX versus gemcitabine for metastatic pancreatic cancer. The New England journal of medicine. 2011; 364:1817-1825.

2. Campbell PJ, Yachida S, Mudie LJ, Stephens PJ, Pleasance ED, Stebbings LA, Morsberger LA, Latimer C, McLaren S, Lin ML, McBride DJ, Varela I, Nik-Zainal SA, et al. The patterns and dynamics of genomic instability in metastatic pancreatic cancer. Nature. 2010; 467:1109-1113.

3. Hidalgo M. Pancreatic cancer. The New England journal of medicine. 2010; 362:1605-1617.

4. Jones S, Zhang X, Parsons DW, Lin JC, Leary RJ, Angenendt P, Mankoo P, Carter H, Kamiyama H, Jimeno A, Hong SM, Fu B, Lin MT, et al. Core signaling pathways in human pancreatic cancers revealed by global genomic analyses. Science. 2008; 321:1801-1806. 
5. Biankin AV, Waddell N, Kassahn KS, Gingras MC, Muthuswamy LB, Johns AL, Miller DK, Wilson PJ, Patch AM, Wu J, Chang DK, Cowley MJ, Gardiner BB, et al. Pancreatic cancer genomes reveal aberrations in axon guidance pathway genes. Nature. 2012; 491:399-405.

6. di Magliano MP, Logsdon CD. Roles for KRAS in pancreatic tumor development and progression. Gastroenterology. 2013; 144:1220-1229.

7. Eser S, Schnieke A, Schneider G, Saur D. Oncogenic KRAS signalling in pancreatic cancer. British journal of cancer. 2014; 111:817-822.

8. Downward J. Targeting RAS signalling pathways in cancer therapy. Nature reviews Cancer. 2003; 3:11-22.

9. Almoguera C, Shibata D, Forrester K, Martin J, Arnheim N, Perucho M. Most human carcinomas of the exocrine pancreas contain mutant c-K-ras genes. Cell. 1988; 53:549-554.

10. Campbell SL, Khosravi-Far R, Rossman KL, Clark GJ, Der CJ. Increasing complexity of Ras signaling. Oncogene. 1998; 17:1395-1413.

11. Corbett KD, Alber T. The many faces of Ras: recognition of small GTP-binding proteins. Trends in biochemical sciences. 2001; 26:710-716.

12. Cullen PJ, Lockyer PJ. Integration of calcium and Ras signalling. Nature reviews Molecular cell biology. 2002; 3:339-348.

13. Scheffzek K, Ahmadian MR, Kabsch W, Wiesmuller L, Lautwein A, Schmitz F, Wittinghofer A. The Ras-RasGAP complex: structural basis for GTPase activation and its loss in oncogenic Ras mutants. Science. 1997; 277:333-338.

14. Lito P, Rosen N, Solit DB. Tumor adaptation and resistance to RAF inhibitors. Nature medicine. 2013; 19:1401-1409.

15. Joseph EW, Pratilas CA, Poulikakos PI, Tadi M, Wang W, Taylor BS, Halilovic E, Persaud Y, Xing F, Viale A, Tsai J, Chapman PB, Bollag G, et al. The RAF inhibitor PLX4032 inhibits ERK signaling and tumor cell proliferation in a V600E BRAF-selective manner. Proceedings of the National Academy of Sciences of the United States of America. 2010; 107:14903-14908.

16. Pratilas CA, Taylor BS, Ye Q, Viale A, Sander C, Solit DB, Rosen N. (V600E)BRAF is associated with disabled feedback inhibition of RAF-MEK signaling and elevated transcriptional output of the pathway. Proceedings of the National Academy of Sciences of the United States of America. 2009; 106:4519-4524.

17. Komander D, Rape M. The ubiquitin code. Annual review of biochemistry. 2012; 81:203-229.

18. Finley D. Recognition and processing of ubiquitinprotein conjugates by the proteasome. Annual review of biochemistry. 2009; 78:477-513.

19. Deshaies RJ, Joazeiro CA. RING domain E3 ubiquitin ligases. Annual review of biochemistry. 2009; 78:399-434.

20. Berndsen CE, Wolberger C. New insights into ubiquitin E3 ligase mechanism. Nature structural \& molecular biology. 2014; 21:301-307.
21. Rotin D, Kumar S. Physiological functions of the HECT family of ubiquitin ligases. Nature reviews Molecular cell biology. 2009; 10:398-409.

22. Li X, Shen L, Zhang J, Su J, Shen L, Liu X, Han H, Han W, Yao L. Degradation of HER2 by Cbl-based chimeric ubiquitin ligases. Cancer research. 2007; 67:8716-8724.

23. Ma Y, Gu Y, Zhang Q, Han Y, Yu S, Lu Z, Chen J. Targeted degradation of KRAS by an engineered ubiquitin ligase suppresses pancreatic cancer cell growth in vitro and in vivo. Molecular cancer therapeutics. 2013; 12:286-294.

24. Hatakeyama S, Watanabe M, Fujii Y, Nakayama KI. Targeted destruction of c-Myc by an engineered ubiquitin ligase suppresses cell transformation and tumor formation. Cancer research. 2005; 65:7874-7879.

25. Zhang J, Zheng N, Zhou P. Exploring the functional complexity of cellular proteins by protein knockout. Proceedings of the National Academy of Sciences of the United States of America. 2003; 100:14127-14132.

26. Caussinus E, Kanca O, Affolter M. Fluorescent fusion protein knockout mediated by anti-GFP nanobody. Nature structural \& molecular biology. 2012; 19:117-121.

27. Portnoff AD, Stephens EA, Varner JD, DeLisa MP. Ubiquibodies, synthetic E3 ubiquitin ligases endowed with unnatural substrate specificity for targeted protein silencing. The Journal of biological chemistry. 2014; 289:7844-7855.

28. Warne PH, Viciana PR, Downward J. Direct interaction of Ras and the amino-terminal region of Raf-1 in vitro. Nature. 1993; 364:352-355.

29. de Rooij J, Bos JL. Minimal Ras-binding domain of Rafl can be used as an activation-specific probe for Ras. Oncogene. 1997; 14:623-625.

30. Leevers SJ, Paterson HF, Marshall CJ. Requirement for Ras in Raf activation is overcome by targeting Raf to the plasma membrane. Nature. 1994; 369:411-414.

31. Bernassola F, Karin M, Ciechanover A, Melino G. The HECT family of E3 ubiquitin ligases: multiple players in cancer development. Cancer cell. 2008; 14:10-21.

32. Peschard P, Kozlov G, Lin T, Mirza IA, Berghuis AM, Lipkowitz S, Park M, Gehring K. Structural basis for ubiquitin-mediated dimerization and activation of the ubiquitin protein ligase Cbl-b. Molecular cell. 2007; 27:474-485.

33. Zhang M, Windheim M, Roe SM, Peggie M, Cohen P, Prodromou C, Pearl LH. Chaperoned ubiquitylation-crystal structures of the CHIP U box E3 ubiquitin ligase and a CHIP-Ubc13-Uev1a complex. Molecular cell. 2005; 20:525-538.

34. Yu X, Yu Y, Liu B, Luo K, Kong W, Mao P, Yu XF. Induction of APOBEC3G ubiquitination and degradation by an HIV-1 Vif-Cul5-SCF complex. Science. 2003; 302:1056-1060.

35. Kibel A, Iliopoulos O, DeCaprio JA, Kaelin WG, Jr. Binding of the von Hippel-Lindau tumor suppressor protein to Elongin B, C. Science. 1995; 269:1444-1446. 
36. Eguchi A, Meade BR, Chang YC, Fredrickson CT, Willert K, Puri N, Dowdy SF. Efficient siRNA delivery into primary cells by a peptide transduction domain-dsRNA binding domain fusion protein. Nature biotechnology. 2009; 27:567-571.

37. Reddy SA. Signaling pathways in pancreatic cancer. Cancer journal. 2001; 7:274-286.

38. Collisson EA, Trejo CL, Silva JM, Gu S, Korkola JE, Heiser LM, Charles RP, Rabinovich BA, Hann B, Dankort D, Spellman PT, Phillips WA, Gray JW, et al. A central role for $\mathrm{RAF} \longrightarrow \mathrm{MEK} \longrightarrow \mathrm{ERK}$ signaling in the genesis of pancreatic ductal adenocarcinoma. Cancer discovery. 2012; 2:685-693.

39. Brummelkamp TR, Bernards R, Agami R. Stable suppression of tumorigenicity by virus-mediated RNA interference. Cancer cell. 2002; 2:243-247.

40. Yuan TL, Fellmann C, Lee CS, Ritchie CD, Thapar V, Lee LC, Hsu DJ, Grace D, Carver JO, Zuber J, Luo J, McCormick F, Lowe SW. Development of siRNA payloads to target KRAS-mutant cancer. Cancer discovery. 2014; 4:1182-1197.

41. Jackson JH, Cochrane CG, Bourne JR, Solski PA, Buss JE, Der CJ. Farnesol modification of Kirsten-ras exon 4B protein is essential for transformation. Proceedings of the National Academy of Sciences of the United States of America. 1990; 87:3042-3046.

42. Berndt N, Hamilton AD, Sebti SM. Targeting protein prenylation for cancer therapy. Nature reviews Cancer. 2011; 11:775-791.

43. Sheehy AM, Gaddis NC, Malim MH. The antiretroviral enzyme APOBEC3G is degraded by the proteasome in response to HIV-1 Vif. Nature medicine. 2003; 9:1404-1407.

44. Kao S, Khan MA, Miyagi E, Plishka R, Buckler-White A, Strebel K. The human immunodeficiency virus type 1 Vif protein reduces intracellular expression and inhibits packaging of APOBEC3G (CEM15), a cellular inhibitor of virus infectivity. Journal of virology. 2003; 77:11398-11407.
45. Shaib Y, Davila J, Naumann C, El-Serag H. The impact of curative intent surgery on the survival of pancreatic cancer patients: a U.S. Population-based study. The American journal of gastroenterology. 2007; 102:1377-1382.

46. Pausawasdi N, Scheiman J. Endoscopic evaluation and palliation of pancreatic adenocarcinoma: current and future options. Current opinion in gastroenterology. 2007; 23:515-521.

47. Sperti C, Moletta L, Merigliano S. Multimodality treatment of recurrent pancreatic cancer: Mith or reality? World journal of gastrointestinal oncology. 2015; 7:375-382.

48. Westerdahl J, Andren-Sandberg A, Ihse I. Recurrence of exocrine pancreatic cancer--local or hepatic? Hepatogastroenterology. 1993; 40:384-387.

49. Sperti C, Pasquali C, Piccoli A, Pedrazzoli S. Recurrence after resection for ductal adenocarcinoma of the pancreas. World journal of surgery. 1997; 21:195-200.

50. Liu C, Zhang X, Huang F, Yang B, Li J, Liu B, Luo H, Zhang $\mathrm{P}$, Zhang H. APOBEC3G inhibits microRNAmediated repression of translation by interfering with the interaction between Argonaute-2 and MOV10. The Journal of biological chemistry. 2012; 287:29373-29383.

51. Ensoli B, Buonaguro L, Barillari G, Fiorelli V, Gendelman R, Morgan RA, Wingfield P, Gallo RC. Release, uptake, and effects of extracellular human immunodeficiency virus type 1 Tat protein on cell growth and viral transactivation. Journal of virology. 1993; 67:277-287.

52. Jensen LB, Torp AM, Andersen SB, Skov PS, Poulsen LK, Knol EF and van Hoffen E. The biological activity of a recombinantly expressed (His)-tagged peanut allergen ( $\mathrm{rAra} \mathrm{h} 1$ ) is unaffected by endotoxin removal. Journal of immunological methods. 2008; 335:116-120. 